\section{'CUSARARE', NUEVA VARIEDAD DE AVENA DE ALTA CALIDAD DE GRANO Y RESISTENTE A ROYAS}

\section{'CUSARARE', A NEW OAT CULTIVAR AND OF HIGH QUALITY GRAIN AND RESISTANT TO RUST}

\author{
José J. Salmerón Z. ${ }^{1 *}$, Víctor Hernández M., \\ Carlos Lara M., Bertoldo Cabañas C., \\ Raymundo Velasco Nuño y Héctor E. \\ Villaseñor M.
}

\begin{abstract}
${ }^{1}$ Programa de Cereales del Campo Experimental Sierra de Chihuahua, del Istituto Nacional de Investigaciones Forestales, Agrícolas y Pecuarias. Hidalgo 1213. 31500 Cd. Cuauhtémoc, Chih. Tel y Fax 01 (625) 258-3110

* Autor para correspondencia (jjsalme@yahoo.com)
\end{abstract}

'Cusarare' es una variedad de avena (Avena sativa L.) desarrollada en el Programa de Mejoramiento Genético de Avena del Instituto Nacional de Investigaciones Forestales, Agrícolas y Pecuarias, que se pone a disposición de los agricultores mexicanos para su siembra en el ciclo otoño-invierno, en condiciones de riego. Esta nueva variedad es de doble propósito: grano y forraje.

Fue aceptada el 27 de febrero del 2007 en el Registro Nacional de Variedades y Plantas con la clave AVE-0013251104, y protegida por la Ley de Producción, Certificación y Comercio de Semillas vigente en México, conforme al reglamento de la Unión Internacional para la Protección de Obtenciones Vegetales (UPOV), para su uso comercial dentro del catálogo de variedades factibles de certificación. Sus ventajas principales son: rendimiento superior a 'Cuauhtémoc' y 'Chihuahua' en 3 y $14 \%$ respectivamente; y resistente a las royas de la corona (Puccinia coronata f. sp. avenae) y del tallo (Puccinia graminis f. sp. avenae). El máximo peso específico del grano es de $499 \mathrm{~g} \mathrm{~L}^{-1}$, satisfactorio para procesar el grano en forma de hojuelas y otros derivados, para consumo humano.

'Cusarare' fue derivada de la cruza: (Yuca/San Carlos//Tulancingo) Diamante "s". El progenitor Yuca procedió de la cruza (Tippecanoe/CI-7114//IndioN/No.58)A6177-C. San Carlos se obtuvo de la cruza (Resist/Putnam 61-CI-7359//Curt)O-Curt. 'Tulancingo' es una variedad comercial que proviene de la cruza (CI-
30034/Tippecanoe//Curt)/ Cuauhtémoc. 'Diamante' es una variedad derivada de la cruza (1955-A-39-3-2//Curt/ Impala) ENA. Para obtener la variedad se siguió el método genealógico o de pedigrí, cuya cruza inicial se hizo en el Campo Experimental Valle de México en 1986; el avance de la generaciones $F_{1}$ a $F_{5}$ se llevó a cabo en el Valle de México, con selección individual de plantas en $\mathrm{F}_{2}$ para resistencia a royas, días a madurez y vigor de planta. A la Sierra de Chihuahua llegó como F6, donde se hicieron cuatro selecciones individuales. Éstas se llevaron a $\mathrm{F}_{7}$ y posteriormente se cosecharon en forma masiva en Winnipeg, Canadá en 1993 y en Calera, Zacatecas en 1994.

A partir de 1996 se hicieron pruebas preliminares de rendimiento en Cuauhtémoc, Chih. y Tepatitlán, Jalisco; luego se establecieron experimentos de evaluación en una distribución en bloques completos al azar con cuatro repeticiones en dos ciclos bajo temporal o secano, en 1997 y 1998 en Chihuahua y Jalisco. En el ciclo otoño-invierno de 1999-2000 se efectuaron evaluaciones bajo condiciones de riego en cuatro localidades de Chihuahua (Cuauhtémoc, Guerrero, Casas Grandes y Delicias), una localidad en Zacatecas y otra en Jalisco. Posteriormente 'Cusarare' se validó en Tepatitlán, Jal. y Delicias, Cuauhtémoc y Asención en Chihuahua, en los años 2002 y 2006, siempre en condiciones de riego.

La resistencia de 'Cusarare' a las royas se evaluó en la etapa de plántula. Para la roya de la corona fue resistente a las siguientes razas: CR13, CR20, CR36, CR50, CR152, CR169, CR185 y CR225. También fue resistente a las siguientes razas de roya del tallo: NA8, NA16, NA25, NA26, NA 27 y NA55. Estos análisis se hicieron en el Centro de Investigación en Agricultura y Alimentos, en Winnipeg, Manitoba Canadá (Salmerón et al., 1996). En pruebas de campo efectuadas en Winnipeg las plantas adultas de 'Cusarare' presentaron resistencia moderada a roya de la corona y susceptibilidad moderada a la roya del tallo (Salmerón et al., 1996). En evaluaciones posteriores en condiciones de campo en Chihuahua, esta variedad presentó resistencia a las razas de roya de la corona: BLBB, NLBQ, QDCQ y JBBL, y a la raza BLBB en Tepatitlán, Jalisco, según la nomenclatura de Chong et al. (2000). En una prueba de 24 ambientes en México, ' $\mathrm{Cu}-$ sarare' fue resistente a la roya del tallo en 23 ambientes, en tanto que las variedades Chihuahua y 'Cuauhtémoc' tuvieron lecturas completamente susceptibles (100S) para las royas del tallo y de la corona, en todos los ambientes (Villaseñor, 2006).

La descripción morfológica de 'Cusarare' efectuada en el Campo Experimental Sierra de Chihuahua durante los ciclos otoño-invierno del 2001 y 2002 indica que la planta 
es de crecimiento intermedio, con leve pilosidad en el margen de las hojas inferiores pero ausente en la hoja bandera. El nudo superior del tallo tiene pilosidad abundante. La panícula posee ramificaciones ordenadas en forma equilátera y de posición semierecta; las espiguillas están en posición colgante. La altura de planta es media,
10 a $15 \mathrm{~cm}$ más corta que la variedad 'Cuauhtémoc' que mide de 110 a $130 \mathrm{~cm}$. El grano tiene cáscara, con aristas débiles que se rompen en la cosecha, y la gluma es de color café. Su tipo de planta y espiga se muestran en la Figura 1.
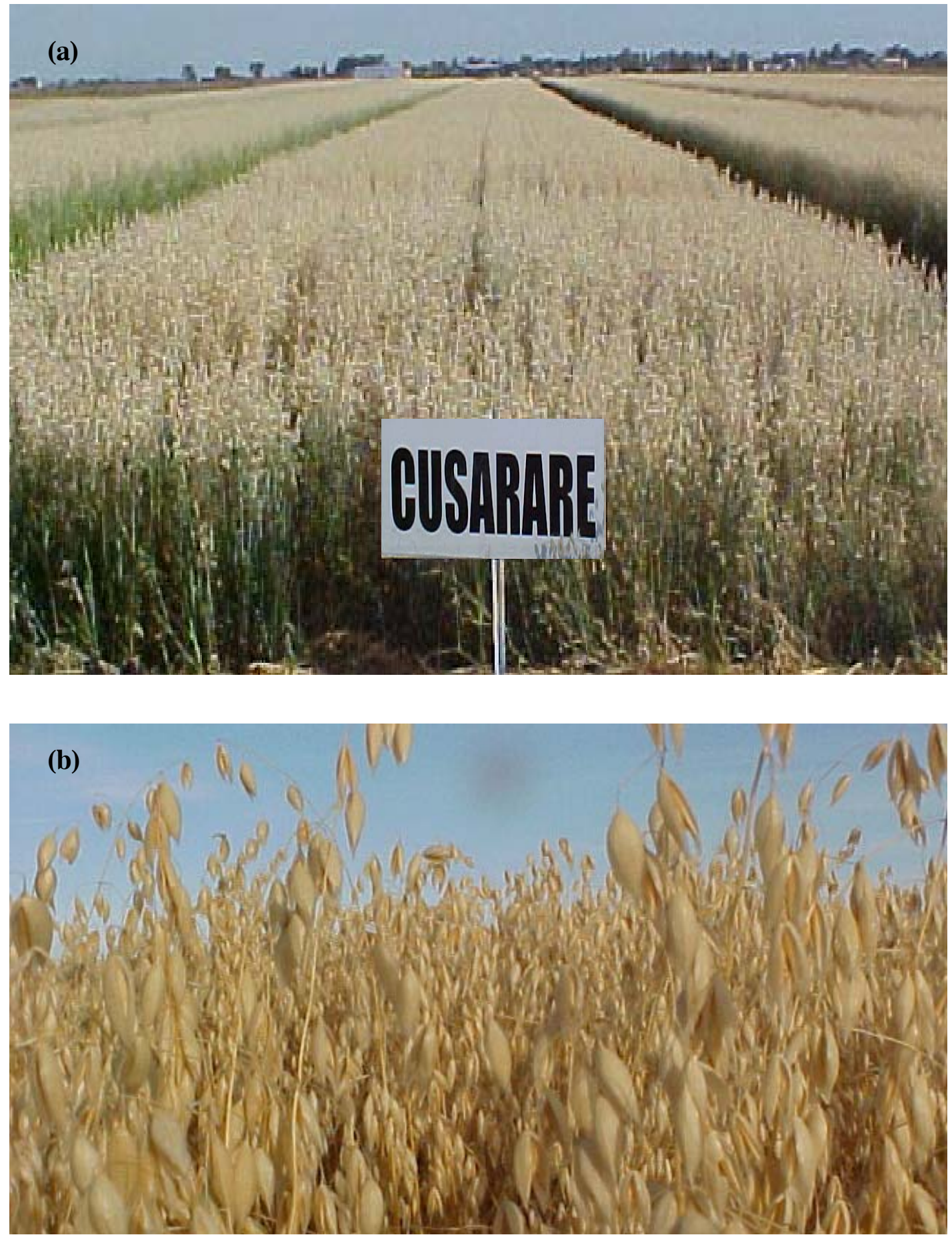

Figura 1. Aspecto de la planta de avena 'Cusarare' (a) y de su espiga (b), en una etapa cercana a la madurez fisiológica. 
En la evaluación de rendimiento de grano hecha en nueve ambientes, 'Cusarare' produjo en promedio 3818 $\mathrm{kg} \mathrm{ha}^{-1}$, que superó a las variedades testigo 'Cuauhtémoc' (3459 $\left.\mathrm{kg} \mathrm{ha}^{-1}\right)$ y 'Chihuahua' (3336 kg ha ${ }^{-1}$ ). En materia seca total medida en tres etapas 'Cusarare' también superó a 'Cuauhtémoc' en 93, 1487 y $130 \mathrm{~kg} \mathrm{ha}^{-1}$ correspondientes a las etapas de embuche, estado lechoso masoso y madurez fisiológica, respectivamente. El porcentaje de proteína en el forraje de 'Cusarare' varió de $22.18 \%$ en la etapa de embuche a $9.94 \%$ en la de madurez, en tanto que 'Cuauhtémoc' fluctuó de 21.9 a $9.66 \%$ para las mismas etapas (Salmerón; Com. personal) ${ }^{1}$. A través de localidades, 'Cusarare' también superó en rendimiento a los testigos 'Cuauhtémoc' y 'Chihuahua' en ambientes de alta producción de forraje, pero en ambientes de bajo potencial 'Cusarare' fue inferior a esos testigos. El peso específico del grano es una característica altamente afectada por el manejo agronómico (fertilización y riegos, principalmente); en esta característica 'Cusarare' fluctuó de 430 a $499 \mathrm{~g} \mathrm{~L}^{-1}$ con una media de $437 \mathrm{~g} \mathrm{~L}^{-1}$, mientras que 'Cuauhtémoc' y 'Chihuahua' tuvieron promedios de 438 y $425 \mathrm{~g} \mathrm{~L}^{-1}$, respectivamente.

Al evaluar la calidad del grano para la industria, ' $\mathrm{Cu}$ sarare' tuvo ventajas sobre las variedades 'Cuauhtémoc' y 'Chihuahua', en: mayores proporciones de grano perlado (sin cáscara) y de grano grande, y menor porcentaje de granos chicos, así como mayor rendimiento industrial (Cuadro 1). La semilla básica de 'Cusarare' para su siembra está disponible en el Campo Experimental Sierra de Chihuahua.

Cuadro 1. Características de calidad industrial del grano (\%), de tres variedades de avena.

\begin{tabular}{lccc}
\hline Características & \multicolumn{3}{c}{ Variedades } \\
\cline { 2 - 4 } & 'Cusarare' 'Cuauhtémoc' & Chihuahua' \\
\hline Grano gordo/doble & 11.73 & 6.37 & 4.11 \\
Grano triple & 3.84 & 0 & 0 \\
Grano perlado & 3.01 & 9.97 & 11.0 \\
Avena liviana y pajilla & 0.75 & 1.29 & 1.61 \\
Avena silvestre & 0 & 0 & 0 \\
Filerillo & 0.14 & 0.50 & 0.94 \\
Grano chico & 9.40 & 12.78 & 9.76 \\
Grano grande & 71.82 & 68.65 & 70.24 \\
Humedad (\%) & 10.21 & 9.6 & 9.6 \\
Rendimiento industrial (\%) & 67.28 & 64.66 & 69.47 \\
\hline
\end{tabular}

\section{AGRADECIMIENTOS}

A Carlos Jiménez González del Programa de Cereales del Campo Experimental Valle de México, por haber proporcionado las poblaciones $\mathrm{F}_{5}$ que incluían las cruzas que

\footnotetext{
${ }^{1}$ Salmerón J J, D Domínguez D, M R Ávila M, R Gutiérrez G (2008) La fertilización com biosólidos em el rendimiento de la avena. Memória del XXI Congreso Nal. De Fitogenética. Tuxtla Gutiérrez, Chiapas. pp:227.
}

generaron a la variedad 'Cusarare'; y a la empresa Avena de Chihuahua S. A. de C. V. por el apoyo financiero otorgado de 1998 a 2000.

\section{BIBLIOGRAFÍA}

Chong J, K Leonard, J J Salmerón (2000) A North American system of nomenclature por Puccuinia coronata f. sp. avenae. Plant Dis. 84:580-585.

Salmerón J J, D E Harder J Chong (1996) Mexican oat germ plasm as a source of resistance to stem rust and crown rust. Plant Dis. 80:404-407.

Villaseñor M H E (2006) Mejoramiento genético y liberación de variedades de avena para la producción de forraje y grano en México. Informe Anual 2005. INIFAP. 57 p. 